\title{
Ovarian, Fallopian Tube, and Primary Peritoneal Carcinoma pT0 TNM Finding v8
}

National Cancer Institute

\section{Source}

National Cancer Institute. Ovarian, Fallopian Tube, and Primary Peritoneal Carcinoma pTO

TNM Finding v8. NCI Thesaurus. Code C139941.

Ovarian, fallopian tube, or primary peritoneal carcinoma with no evidence of primary tumor. (from AJCC 8th Ed.) 